\title{
Processing redundant signals: Coactivation, divided attention, or what?
}

\author{
CHARLES W. ERIKSEN, BARRY GOETTL, JAMES D. ST. JAMES, and LISA R. FOURNIER \\ University of Illinois, Urbana-Champaign, Champaign, Illinois
}

\begin{abstract}
The evidence for and against a redundancy gain in reaction time (RT) when the target is repeated in the visual display is reviewed. We consider the relevance of redundancy gains under these circumstances to the question of whether attention can be simultaneously directed to separate locations in the visual field. In the present experiments, two capital letters were the target stimuli in a two-alternative forced-choice RT paradigm. In addition to the usual conditions of single-target trials, trials on which the target is repeated in the display, and trials on which the target occurs with a noise letter, we introduced the innovation of a condition in which both targets occur in the display. In our two experiments, RT was fastest with single-target displays and slowest with displays containing a target and a noise letter. There was no significant difference in RT to displays in which the target was repeated and displays in which both targets were presented. Both conditions showed a redundancy gain when compared with displays containing a target and a noise letter. The lack of response competition in the both-targets condition and the overall pattern of the results were well explained by a unitary attentional focus that serially processed the letters in the display. Analyses of minima and maxima RTs were consistent with this interpretation.
\end{abstract}

In the present research, our major purpose was to determine the role of focal attention in the redundant-signals effect as it applies to visual recognition. This effect refers to the gain in recognition accuracy or the decrease in discriminated response latency that sometimes occurs with redundant-target presentations. For example, on a twochoice response time (RT) task, the subject may be required to discriminate between the targets A and B. A redundant-signals effect is obtained if the average $R T$ is shorter when two As or two Bs are presented simultaneously on each trial than when a trial consists of only a single $\mathrm{A}$ or $\mathrm{B}$.

Although redundancy of signals can be presented across separate sensory modalities or within any modality, in the present paper, we will be concerned only with visual signals. Specifically, we have studied the effect upon recognition latency for targets when they occur once as opposed to twice in the visual field on a trial.

The presence or absence of a redundant-signals effect or its magnitude, if present, has important implications about basic characteristics of information processing. Not only is it relevant to issues of serial as opposed to parallel processing of inputs, but it also bears upon questions of whether we can simultaneously divide the focus of attention to separate sensory modalities or to separate locations in the visual field. Mullin, Egeth, and Mordkoff (1988) have suggested that the redundant-signals paradigm

This research was supported by Public Health Service Research Career Program Award K6-MH-22014 to the first author and by United States Public Health Service Research Grant MH-01206, also to the first author. Barry Goettl is now at Clemson University. Requests for reprints may be sent to Charles W. Eriksen, Department of Psychology, University of Illinois, Urbana-Champaign, 603 E. Daniel, Champaign, IL 61820. might be useful in resolving certain issues regarding serial versus parallel processing. They note that there are apparently no serial models that can predict a redundancy gain. Unfortunately, though, there are a number of problems that arise in attempting to determine whether a genuine redundancy gain has been achieved.

When the dependent variable is recognition accuracy, the weight of evidence shows that a redundancy gain occurs when multiple instances of the target are simultaneously presented in the visual display (Doherty \& Keeley, 1969; C. W. Eriksen, 1966; C. W. Eriksen \& Lappin, 1965; Keeley \& Doherty, 1971; Kinchla, 1974). Experiments using accuracy as a dependent variable, however, are not capable of answering questions addressing either serial versus parallel processing or the possibility of dividing focal attention. The reason is that the subjective duration of a visual stimulus is not the same as the stimulus' physical duration, and the icon has been shown to persist for as much as several hundred milliseconds after the termination of the physical stimulus (C. W. Eriksen \& Collins, 1969; Sperling, 1960). Di Lollo (1977) has recently presented evidence that even extremely brief visual stimuli have a subjective duration on the order of $80-120 \mathrm{msec}$. With the stimulus trace lasting as long as $100 \mathrm{msec}$, there is sufficient time for attention to switch from one stimulus to another or for serial processing of at least two or three simultaneous inputs. This would permit identification to be based upon the clearest of the separate inputs or, perhaps, for information to be pooled over the partial cues contained in each of the inputs (Doherty \& Keeley, 1969; Keeley \& Doherty, 1971).

The above problem is avoided when response latency is measured. Experiments employing latency measures 
most generally have obtained a redundancy gain, but there is considerable controversy over how much of a gain is achieved and whether the gain is artifactual. On a trial with redundant targets, serial processing of separate stimuli would lead to a response as soon as the first stimulus completed processing. Continuing to process the remaining targets would essentially be a waste of time, since a response would have already been initiated. Serial processing could not lead to a redundancy gain. If, however, the separate stimuli were parallel-processed, the first stimulus to complete processing or reach a response criterion would activate the response. If the processing time for each of the targets has a random duration and these distributions overlap, then, on the average, RT will be faster with two targets than it will with one. There would be a race between the separate processing of the two stimuli, and the winner would activate the response. On the average, the time for the winning process would be less than the time for either process alone. Raab (1962) termed this redundancy gain statistical facilitation.

While each of the two inputs may be separately and simultaneously processed, both could be affected by a common variable that leads to either positive or negative correlation in their rate of processing. Variation of the subject's motivational level over trials or variation in response criterion could effect the separate-stimulus processing on a given trial. Even such events as eye blinks or uncontrolled noises in the laboratory could introduce correlation. While investigators (e.g., C. W. Eriksen, 1966; Meijers \& Eijkman, 1977) have concluded that redundancy gains fit a model of statistical independence, it seems more reasonable that some correlation would exist. Indeed, Van der Heijden, Schreuder, Maris, and Neerincx (1984) not only found correlation in the processing of redundant stimuli, but also found the magnitude and direction of the correlation to vary for trials with slow, medium, and fast RTs. Grice, Canham, and Buroughs (1984) also found significant correlations in the processing of redundant stimuli.

Not only is parallel processing, or separate activation, consistent with correlation in the processing of the separate inputs, but, as Miller (1982a) has pointed out, there is the possibility that coactivation can occur with redundant signals. With coactivation, separate and simultaneous processings occur for the individual stimuli, but the output of these separate processings is fed continuously to a common response criterion. With pooling of activation from the separate processings, responses can be faster than any responses elicited by single-stimulus presentations.

Miller (1982a) has proposed a rather simple but effective test for choosing between separate-activation and coactivation models of the redundancy gain. It is based upon the cumulative density functions (CDFs) for the response latencies obtained for the single-stimulus trials and for the redundant-target trials. With separate activation, response latency $(t)$ to redundant targets is determined by the fastest of the individual processes. Thus,

$$
\begin{aligned}
\mathrm{P}(\mathrm{RT})<t / \mathrm{T} 1 & +\mathrm{T} 2) \\
= & \mathrm{P}(\mathrm{RT}<t / \mathrm{T} 1)+\mathrm{P}(\mathrm{RT}<t / \mathrm{T} 2) \\
& -[\mathrm{P}(\mathrm{RT}<t / \mathrm{T} 1)+\mathrm{P}(\mathrm{RT}<t / \mathrm{T} 2)]
\end{aligned}
$$

This holds for all values of $t$. The left term of the expression is the CDF for the redundant-target trials, and the first two terms on the right are the CDFs for the two kinds of single-target trials. The last term is a form of correlation between the separate activation of the two simultaneously presented targets. Miller points out that since the last term in the equation is equal to or greater than zero, it follows that with separate activation, for all values of $t, \mathrm{P}(\mathrm{RT}<t / \mathrm{T} 1+\mathrm{T} 2)$ is less than or equal to $\mathrm{P}(\mathrm{RT}<$ $t / \mathrm{T} 1)+\mathrm{P}(\mathrm{RT}<t / \mathrm{T} 2)$. Thus, separate-activation models require that the probability of $\mathrm{RT}$ smaller than some value of $t$ obtained with redundant targets cannot exceed the sum of the probabilities obtained with single-target presentations. If this inequality is violated, separate-activation models can be rejected. Violations, however, are consistent with coactivation, since RTs to redundant targets can be faster than the fastest RT to a single target.

Miller (1982a) carried out several experiments with redundant targets, using intermodal presentation and experiments in which single and redundant target letters were presented in visual displays. He concluded that, in terms of his proposed test, there was evidence that coactivation occurred not only intermodally but also in vision.

Miller's conclusions with respect to the visual displays have been criticized by Grice, Canham, and Buroughs (1984) and Van der Heijden et al. (1984). Both papers note the evidence for coactivation with visual targets was slight and inconsistent. Grice et al. (1984) further point out that Miller's data for single-target presentation were obtained when the target was presented with a noise letter in the visual display. This offered the opportunity for a response-competition effect (B. A. Eriksen \& C. W. Eriksen, 1974) to increase RTs for the single-target trials. Since there were no potentially response-competitive noise letters in the display when redundant targets were presented, the inflated RTs for the single trials would lead to underprediction of the RTs to be expected for separate activation with the redundant presentation.

Grice, Canham, and Gwynne (1984) used two targets in a choice RT task with displays that contained a single target letter, displays that contained a target letter and a neutral noise letter, and redundant displays that contained the target and repetition of the target. RT was fastest with displays containing a single letter. A redundancy gain was achieved only when the redundant displays were compared with displays containing a single target and a noise letter. The experimenters interpreted these results in terms of a distraction decrement. The presence of two letters in the display constitutes a distraction that reduces the strength of the target letter. This distraction effect apparently is sufficient to override a redundancy gain. However, if the redundant displays are compared with 
the target and noise displays, the two-letter distraction effect is balanced, and then the effect of redundancy can be observed. As noted above, there was a redundancy gain with this comparison, and, when Miller's test was applied to the data, coactivation was indicated. Both targets appear to have contributed their strength equally to the response.

There is evidence in support of a distraction decrement that comes from other choice RT experiments that were not investigating the redundancy effect. C. W. Eriksen, Hamlin, and Daye (1973) and C. W. Eriksen and Schultz (1978) found that such irrelevant stimuli as digits, geometric forms, or even splotches of colors that were presented in the visual field along with target letters impaired discriminatory RT to these letter targets. More recently, Treisman, Kahneman, and Burkell (1983) and Kahneman, Treisman, and Burkell (1983) have reported similar effects.

Van der Heijden et al. (1984) failed to find a distraction effect in their experiments. The stimuli again were letters, but instead of choice RT, they employed a go/nogo task. Subjects pressed response buttons to displays containing one or two Es and refrained from responding when one or more Os appeared. Average RT to displays with two Es was appreciably faster than that for single target displays, but no significant violation of the Miller inequality was obtained. Instead, the experimenters interpret their results as supporting separate activation with negative correlation between the separate channels on trials with fast RTs and positive correlation when response times were longer. Thus, the issue of whether separate activation or coactivation best describe the processing of redundant targets is at best unclear. In fact, in view of the Grice, Canham, and Gwynne (1984) failure to obtain faster RTs to redundant targets than those to single targets presented alone in the display, the very issue of redundancy gains is somewhat questionable.

The issue of separate activation versus coactivation most likely cannot be resolved by applying the Miller inequality. While violation of the inequality would constitute clear evidence for coactivation, the existence of such factors as correlation between the channels processing the separate stimuli and the distraction effect with increases of stimuli in the visual display would reduce the likelihood that the inequality would be violated, even if there was pooling of the output from the processing of the separate targets. Furthermore, as C. W. Eriksen (1988) has pointed out, the Miller inequality fails to take into account response errors. Errors arising from fast guesses, in particular, would bias the Miller test against the finding of coactivation. A more promising approach to the question of coactivation would be in terms of experimental manipulations, and one purpose of the present experimentation was to test for coactivation by manipulating certain variables.

Before considering the experimental design of the present research, however, we must consider the relevance of the problem of focused versus divided at- tention to redundant targets. The parallel processing of separate targets that is presumed by both separateactivation and coactivation models poses no problem for late-selection theories of attention (see, e.g., Duncan, 1980; Hoffman, 1979). Late-selection theories assume quite detailed stimulus processing at a preattentive stage that is of large, if not of unlimited, capacity. For separate activation, a race between the redundant stimuli would determine which stimulus would be available for selection into Stage 2 for further processing or for response. Coactivation also would expedite the processing in the preattentive stage to make the stimulus available for selective entry into Stage 2.

Early-selection models of attention, on the other hand, are much more intimately involved with a possible redundancy effect in visual displays. These models ascribe limited stimulus processing to the preattentive stage. Instead, focal attention is required for conjoining dimensions (Treisman \& Gelade, 1980) or for stimulus identification (Posner, Snyder, \& Davidson, 1980). The separate identification of redundant targets would then seem to require both that focal attention can be divided and that attention can be focused simultaneously to two or more locations in the visual field. Posner et al. (1980) and others (e.g., Broadbent, 1982) have used the spotlight analogy for visual attention, with the additional assumption made that even stimulus detection requires the attentional spotlight (Posner et al., 1980; Shulman, Remington, \& McLean, 1979). Posner et al. have further concluded that the attentional spotlight cannot be divided, and the results of several other experimenters have failed to obtain evidence that focal attention can be simultaneously directed to separated locations in the visual field (C. W. Eriksen \& Yeh, 1985; Jonides, 1983). However, in considering the question of divided attention, attention theorists have seldom considered the evidence that comes from the redundant-targets paradigm.

There are several possible ways that a redundancy gain could be reconciled with early-selection models of attention. One is to deny that the gain comes from separateactivation or coactivation processes. There is ample evidence that an attentional focus can be localized in the visual field (e.g., Murphy \& C. W. Eriksen, 1987); in fact, by precuing procedures, the focus can be directed to spatial location before stimulus presentation, with a consequent expediting of stimulus processing. In redundanttarget paradigms, the possible locations of the targets in the visual field are known to the subject before the trial begins. It could be assumed that the subject, prior to a trial, focuses his/her attention on one of the two possible locations. On the average, the subject will have selected the correct location on half of the trials. When he/she is correct on location, the target will be expedited in processing. When he/she is wrong, some time might be assumed to be needed to focus attention on the target that has $\mathrm{oc}-$ curred in the alternate location. On redundant-target trials, whichever posotion the subject has prefocused will be correct. Thus, on redundant trials, one target will always have 
the gain in processing speed. Other things being equal, the average gain will be equal to one half of the time required to refocus attention from the wrong location to the location where the target appeared.

This possibility has been considered by Van der Heijden et al. (1984), and the data in their experiment were analyzed for this possible effect. Indeed, just such a predicted effect was present in the data, but when a correction was made for pretrial focusing of attention, there was still a significant redundancy gain, which the experimenters attributed to separate activation. Mullin et al. (1988) have recently simulated the effect of prefocusing attention on one or the other of possible target locations, demonstrating that an appreciable performance gain could be obtained with target redundancy. They also explored several data analyses that could be used to separate the attentional effect from true redundancy gains arising from parallel processing of the redundant stimuli.

Separate activation and coactivation can also be reconciled with early-selection models by assuming that the redundancy gain occurs only when the targets represent easy or very well-learned discriminations. There is evidence that visual attention can operate in a distributed mode as well as in a focused mode (C. W. Eriksen \& Yeh, 1985; Jonides, 1980, 1983). Furthermore, a number of experiments have found evidence of parallel processing of visual stimuli when the discrimination involved is gross or the task has been well practiced with consistent mapping of targets and background noise (C. W. Eriksen \& Spencer, 1969; Gardner, 1973; Kinchla, 1974; Schneider \& Shiffrin, 1977; Shiffrin \& Gardner, 1972; Shiffrin \& Schneider, 1977). A test of this explanation could be made by varying the difficulty of the discrimination required and determining whether the redundancy gain varied correspondingly. It might be found that a redundancy gain is obtained only for stimuli for which automatic processing occurs. With discriminations that require focused attention, no redundancy gain would be expected over that attributable to bias in the pretrial attentional focus.

Perhaps the most straightforward manner of accommodating separate-activation and coactivation evidence would be for attention models to permit attending to more than one spatial location or stimulus at the same time. Current conceptions of attention as processing energy (Jonides, 1983; Kahneman, 1973; M. L. Shaw, 1978) are particularly amenable to division of attention over several locations or stimuli. M. L. Shaw (1978) developed a model in which attentional resources can be simultaneously allocated to different display positions in terms of the a priori probability of target occurrence. Results from the experiments show that both recognition accuracy (M. L. Shaw \& P. Shaw, 1977) and RT to targets appearing in these different locations (M. L. Shaw, 1978) correspond to the a priori probability of target occurrence in a location. Unfortunately, the results are equivocal as to whether attention is allocated to different locations and in differing amounts on each trial. Since the data are averaged over trials, comparable results could be obtained if the subjects were probability matching over trials. They could be varying the position to which they direct a unitary attentional focus in keeping with the a priori probabilities of target occurrence. C. W. Eriksen and Yeh (1985) and Jonides (1980) have obtained results that show that subjects do match probabilities over trials.

An alternative to partialing out attentional resources to different locations in the visual field is to conceive of the attentional focus as varying in size. C. W. Eriksen and Yeh (1985) and C. W. Eriksen and St. James (1986) have proposed a zoom lens model of visual attention in which the size of the attentional focus can expand or contract. Like with a zoom lens, when the attentional field is large, there is less processing or resolution of detail than there is when the field is contracted. As attention zooms in on a stimulus, there is enhanced processing of detail, but at the cost of reducing the number of stimuli within the field that receives processing. Applied to the redundant-targets paradigm, parallel processing of the redundant targets would occur if the attentional field was enlarged to encompass all possible target locations. However, since detail resolution is lost when the field expands, the discriminatory requirement of the task could not exceed the resolving power of the expanded attentional field.

Both the zoom lens conception and the spatial allocation model of M. L. Shaw (1978) encounter difficulty in accounting for redundancy gain. As the number of positions or the size of the field is enlarged, the amount of attentional energy or resources available to each position or stimulus within the field is assumed to decrease. Thus, if two targets are presented, the amount of resources devoted to each would be less than if only a single target was presented. As a consequence, the speed of processing each target on redundant trials would be less than when only a single target was presented. Attempts to predict the RT distribution for redundant trials using the distributions obtained for single target trials would be inappropriate. The Miller inequality would never be violated. In fact, even if both targets were processed in parallel, there might be no evidence of redundancy gain, since the loss of processing efficiency or speed with divided resources might be greater than any gain achieved by separate activation or even coactivation.

As noted above, Grice, Canham, and Gwynne (1984) found that when the target was presented twice in the display, the average RT was greater than the average RT for single targets presented alone. Evidence for a redundancy gain was obtained only when the RT for redundant trials was compared with single targets that were presented with a neutral noise letter. Grice, Canham, and Gwynne attributed this finding to a distraction decrement that occurs with an increase in the number of stimuli in the display, but it may also indicate the operation of some of the attentional factors we have considered above. The distraction decrement may arise from the sharing of attentional resources between the two stimuli in the field. As a result, each of these stimuli is processed slower on the average than when all available resources are devoted to 
a single stimulus. Even if coactivation occurs, the gain derived from it is still less than the loss of processing speed resulting from the sharing of resources. However, the procedure used by Grice, Canham, and Gwynne may be a solution to determining whether separate activation or coactivation is occurring. The comparison should not be between a single-stimulus field and the redundant condition, but rather between a two-stimulus display in which only one is a target and a two-stimulus display in which both are targets.

The distraction decrement could also arise from an indivisible attentional focus. When only a target is presented in the visual display, the focus is directed to the target. But when a target and a neutral noise letter are presented, on half of the trials, the subject can be presumed to direct the focus to the noise letter first. Some time would be required for the processing of the noise letter, to the extent that it would be recognized as a nontarget, and then attention would be shifted to the other stimulus in the field. As a consequence, RT to a display and a noise letter would average longer than it would for target-alone displays. However, a redundancy gain would be obtained for the two-target displays relative to the target and noise-letter displays, since, in the former, no matter to which stimulus the subject first directed his/her attentional focus, a target would be processed. Other factors equal, performance with the redundant displays should be at the level obtained for single-target displays.

In the present research, we have approached the redundant-signals problem from the point of view of attention theory. We have attempted to determine what happens when two separated locations in the visual field are cued or precued in a multiletter display. Do both locations show evidence of enhanced processing consistent with the division of the attentional focus, or does the evidence indicate that the subjects focus attention on only one indicated position on a given trial? We believed we could answer these questions and avoid the problem of subjects' probability matching over trials by the use of a response-competition paradigm (B. A. Eriksen \& C. W. Eriksen, 1974; C. W. Eriksen \& Schultz, 1979).

\section{EXPERIMENT 1}

The tasks were two-choice RT with the target letters $\mathrm{S}$ and $\mathrm{C}$. The target letter (or letters) was imbedded in a circular display of eight letters. On each trial, two locations were either precued $150 \mathrm{msec}$ before display onset or cued simultaneously with display onset. The cue consisted of an underlining of the display position. Although the display letters were evenly distributed around the circular arrangement, target location was restricted to the four locations corresponding to the clock positions of $3,6,9$, and 12 o'clock. On $80 \%$ of the trials, only one of the cued locations contained a target letter, with the other cued location containing one of the noise letters $(\mathrm{H}, \mathrm{K}, \mathrm{N}, \mathrm{V}, \mathrm{W}$, or $\mathrm{Z})$. On $10 \%$ of the trials, both cued locations contained the same target letter (identical- targets condition), and on the remaining $10 \%$, one cued location contained one of the target letters and the other location the other target letter (both-targets conditions). To forestall possible confusion on the part of the subject if he/she saw both target letters, the subject was informed that, if this circumstance should arise, he/she was to respond to the target letter seen first or to the one that seemed clearest.

The critical experimental manipulation was the condition in which both target letters occurred in the cued locations. If the subject was focusing attention simultaneously on both locations, we anticipated that RT would appreciably increase due to response competition. A considerable volume of research has shown that, in twochoice RT tasks, response latency is appreciably increased if the target stimulus is accompanied or flanked by the alternative stimulus (e.g., Coles, Gratton, Bashore, C. W. Eriksen, \& Donchin, 1985; B. A. Eriksen \& C. W. Eriksen, 1974; C. W. Eriksen \& Shultz, 1979; Flowers \& Wilcox, 1982; Grice, Canham, \& Schafer, 1982). Thus, simultaneously attending both cued locations should facilitate the processing of both targets, with a resulting strong response-competition effect relative to the trials on which one of the cued locations contained a neutral noise letter. On the other hand, on those trials in which both cued locations contained the same target, no response competition would be expected, since both cued locations primed, or activated, the same response.

\section{Method}

Subjects. Two men and 2 women, students at the University of Illinois, Urbana-Champaign, served as paid volunteers. All were right-handed and had normal or corrected-to-normal vision.

Apparatus and Stimuli. The stimuli were presented in a threefield Scientific Prototype model GA tachistoscope, equipped with Sylvania FT5/CWX fluorescent lamps. Luminance in all three fields was maintained at $9 \mathrm{fL}$, as measured by a Spectra spot photometer. The subject initiated the onset of trials by pressing a button held freely in the left hand. This triggered the tachistoscope and also a Lafayette digital timer (model 54417). A handrest, located to the right of the subject, contained a small lever that could be moved either right or left, depending upon the target identified by the subject. When the subject moved the lever in either direction, the timer was stopped, and a light located below the timer indicated which response the subject had made. RT was recorded in milliseconds.

The target and noise letters were all black uppercase letters selected from Zip-a-Tone Futura Bold 20-point transfer lettering. Each letter subtended $.25^{\circ}$ visual angle in height. The letters were presented on white vinyl cards.

For each display, eight letters were arrayed on an imaginary circle, $3^{\circ}$ of visual angle in diameter. All displays always contained the target letters $S$ and $C$, with the noise letters $H, Z, K, N, V$, and $W$ randomly ordered between the target positions. Four of the letters were in positions corresponding to clock locations 12,3 , 6 , and 90 'clock. The other four letters were evenly spaced between these.

The targets, $S$ and $C$, were restricted to appear at the $3,6,9$, and $12 o^{\prime}$ 'clock positions. Each target letter occurred equally often in each of the four possible target locations. Six cue cards (clear plastic) were constructed with black bars (lower case 1s from the Zip-a-Tone set), appearing on the extension of the radius through 
the above-listed clock positions, the bar beginning $.25^{\circ}$ of visual angle beyond the letters. Combining the cue cards with the letter cards, it was possible on each trial to indicate either one target letter and one noise letter or two target letters. Note that both target letters occurred on every display, but only on the both-targets conditions were both target letters indicated. On the identical-targets trials, the two indicated locations contained the same target. On the single-target trials, one of the targets and one of the noise letters in a possible target position were cued. In the both-targets condition, both the $S_{i}$ and the $C$ were cued, but one of the remaining nontarget positions also contained either an $\mathbf{S}$ or a $\mathrm{C}$. A similar procedure was employed on identical-target trials. Thus, if $S$ was indicated or cued in two locations, a C occurred in one of the nonindicated target locations. This practice of including a noncued target letter among the noise background was employed to ensure that the subjects used the cues in processing the display.

Fixation was provided by a thin + subtending $25^{\circ}$ of visual angle, located in the center of the imaginary circle. On half of the blocks of trials, the cues appeared $150 \mathrm{msec}$ before the letter display. These cues were placed in Field 2 of the tachistoscope, with a white vinyl card behind them for contrast. On the other half of the blocks of trials, the cues appeared simultaneously with the letter display and were placed in the same field (Field 1). When the cue card was in Field 2, a clear card was placed over the letter display to reduce contrast to equal that of the simultaneous condition. During the simultaneous blocks, a blank clear card over a blank white card was placed in Field 2, so that the 150-msec interval contained an equivalent amount of light stimulation but no location information. In both leading- and simultaneous-cue conditions, the letter display was on for $50 \mathrm{msec}$.

Procedure. The subjects were instructed to initiate each trial by pressing a pushbutton held in the left hand when the fixation point was in clear focus and to keep their face firmly against the viewing hood at all times. A small lever, held with the thumb and forefinger of the right hand, was used to make the response. Further instructions to the subject were: "In this study, you will be seeing displays of eight letters of the alphabet, appearing in a circular arrangement. Two of the letters, the $C$ and the $S$, are your target letters. On each trial, two cue lines will point to locations of the letters, and one of these cues will be pointed to either a $\mathrm{C}$ or an $S$. You are to move the lever to the right if it is a $C$, and to the left if it is an S. [Half of the subjects received the opposite assignment.] There is an exception to the above. On $20 \%$ of the trials, each of the cues will point to a target. When this happens, you are to respond to whichever one you see most clearly or whichever one you see first."

The subjects were told to respond quickly but with only a few errors per block of 60 trials. Each subject took part in one full practice session and four experimental sessions of four blocks of 60 trials each. All blocks contained an equal number of trials on which $\mathrm{S}$ and $\mathrm{C}$ were targets, and each combination of letter and position was cued equally often. Also, in each block, there was an equal number of trials from the identical- and both-targets conditions. Within blocks, targets and positions were presented randomly, with the restriction that neither one target nor one position be cued more than four times in a row. The order of block presentations (leading or simultaneous cues) was counterbalanced across sessions.

\section{Results}

The significance of the results was evaluated in a withinsubjects design for the variables single versus double targets, simultaneous versus precuing, and whether the cued displayed locations were separated by one or three display positions. The effect for number of targets was significant beyond the .01 level $[F(1,3)=56]$, as was the effect for precuing $[F(1,3)=34]$. Spatial separation be- tween cued locations did not approach significance $(p<$ .30 ). There was a significant interaction $[F(1,3)=27$, $p<.02]$ between single versus double targets and whether the locations were cued simultaneously or $150 \mathrm{msec}$ before display presentation. No other interaction approached significance.

Differences between the identical- and both-targets conditions were evaluated in a second ANOVA (subjects, identical vs. both targets, and method of cuing). The effect for target conditions did not approach significance $(p<.20)$, but the method of cuing again was significant $(p<.05)$. None of the interactions approached significance.

The mean RTs for correct responses are shown in Table 1 for the single-target, identical-targets, and bothtargets conditions for simultaneous cuing and precuing $150 \mathrm{msec}$ before display presentation. For both the simultaneous and precue conditions, the latency for single targets was appreciably longer than it was for either of the double-target conditions. However, there was little difference in the RTs between the two types of double targets. For simultaneous cuing, RTs were $13 \mathrm{msec}$ longer to both targets than RTs to identical targets, but, with precuing, the difference was $11 \mathrm{msec}$ in favor of the both-targets condition. Thus, there is no consistent evidence that RTs were delayed by response competition when both targets were cued in the display.

Displays containing two cued targets, whether repetitions of the same target or presentation of both targets, were responded to faster than were displays with only one target in a cued location. The advantage of double targets was somewhat less when the locations were precued, 31 versus $43 \mathrm{msec}$.

The response latency advantage of double targets was consistent with a unitary focus of attention in which the subjects focused first on one cued location and, if the target letter was not there, reoriented their attentional focus to the other cued location. On single-target trials, if the subject focused first on the incorrect location, he/she had to process the noise letter to the point where a decision could be made that the location did not contain a target. Attention then had to be reoriented to the other cued location. Processing a noise letter and then reorienting attention can be presumed to require time.

C. W. Eriksen and Yeh (1985), in an experiment using comparable displays, obtained estimated values of about $70 \mathrm{msec}$ for the time required to process an incorrect location and then reorient attention. If we assume that

Table 1

Mean Response Times (in milliseconds) for Single- and Double-Target Conditions Obtained with Simultaneous Cues and 150-msec Precues

\begin{tabular}{lcccc}
\hline & \multicolumn{2}{c}{ Target Conditions } & \\
\cline { 2 - 3 } & $\begin{array}{c}\text { Single Target } \\
\text { and Noise Letter }\end{array}$ & $\begin{array}{c}\text { Identical } \\
\text { Targets }\end{array}$ & $\begin{array}{c}\text { Both } \\
\text { Targets }\end{array}$ \\
\hline Simultaneous Cue & 559 & 510 & 523 \\
150 -msec Precue & 531 & 505 & 494 \\
Combined Mean & 545 & 508 & 509 \\
\hline
\end{tabular}


this reorientation was required on half of the single-target trials in Experiment 1, then we obtain estimates of 90 and $70 \mathrm{msec}$ from the simultaneous and precued conditions, respectively. These values are in reasonable agreement with those obtained by C. W. Eriksen and Yeh (1985).

If the above account was correct, we would expect the response latency distributions obtained for the single-target trials to be a mixture of two underlying distributions. There would be a distribution for those trials on which the subject found the target in the location that was focused upon first, and a second distribution for those trials where a change of attentional focus was necessary. The difference in the means of these distributions would be in the range of $70-90 \mathrm{msec}$, but most importantly, the composite distribution would have the same minimus as the distribution arising from the double-target trials. The minimus obtained from the single-target trials would correspond to those trials on which the subject focused on the correct target location first. With double-target trials, the subject always focused on the correct location first.

For each subject, the fastest $5 \%$ of RTs were determined for the single- and double-target conditions under both methods of cuing. The means for these minimal RTs are shown in Table 2 . Under both methods of cuing, the means for the single- and double-target conditions are quite similar. A $t$ test of the largest difference, which occurred with the precue, did not approach significance $(p>.20)$; thus, the data are consistent with a serial or sequential search of the cued locations.

A question can be raised as to whether these minimal RTs obtained from each subject's distributions represent "fast guesses." If this were the case, then the finding that the minimal RTs were the same under the single- and double-target conditions would be uninformative as to whether the distribution of RTs to the single-target trials were a mixture of two distributions. There are two lines of evidence that indicate that these minimal RTs reflect the same processing behaviors that underlie the longer RTs. Note that in Table 2 the means reflect the same differences between simultaneous and precuing methods as did the means based upon the entire correct RT distributions. This is not consistent with an interpretation that the minimal RTs are fast guesses.

A second line of evidence comes from examining the RTs on the error trials. If there were fast guesses, the error trials should be composed, in large part, of the fast guesses that were wrong (a priori probability $=+.5$ ).

\section{Table 2}

Mean of the Minimal Response Times (in milliseconds) for Single- and Double-Target Conditions Obtained with Simultaneous Cues and 150-msec Precues

\begin{tabular}{lcc}
\hline & \multicolumn{2}{c}{ Target Conditions } \\
\cline { 2 - 3 } & $\begin{array}{c}\text { Single Target } \\
\text { and Noise Letter }\end{array}$ & Double Targets \\
\hline Simultaneous Cue & 382 & 381 \\
150-msec Precue & 372 & 361 \\
Combined Mean & 377 & 371 \\
\hline
\end{tabular}

For the single- and identical-target conditions, errors occurred on $6 \%$ of the trials. The mean RT for errors with simultaneous cuing was $582 \mathrm{msec}$, and for precuing $524 \mathrm{msec}$. These means were larger than the means obtained for these cuing methods on correct trials and were over $150 \mathrm{msec}$ slower than the comparable means of the minimal RT trials.

Like the minimal RTs, the error-trial latencies also reflect the effects of the experimental manipulations. ${ }^{1}$ The mean error-trial latency was significantly longer for simultaneous cuing than it was for precuing $[t(3)=2.99$, $p<.05$, one-tailed]. Thus, there is no support for the argument that fast guesses are the basis for the singleand double-target RT distributions having essentially the same minima.

The finding that the mean RTs on error trials were longer than the mean latencies for correct responses is important in another context: It disambiguates the comparison between the mean latencies of the identical- and both-targets conditions. In the identical-targets condition, it was possible for the subjects to make errors, as in the single-target condition. However, in the both-targets condition, since both targets were present in the display, there technically was not an incorrect response. Thus, no errors occurred in the latter condition. If it had developed that the mean latency for errors was appreciably shorter than that for correct responses, the failure to find a difference between the identical- and both-targets conditions could have been attributed, at least in part, to fast undetectable errors in the both-targets condition. However, the finding that the mean latency for errors was actually greater than that for correct responses precludes this possibility. This conclusion, of course, is based upon the reasonable assumption that the undetectable errors in the both-targets condition had the same basis, or causes, as they did in the identical-targets condition.

The mixture distribution interpretation of the singletarget trials also requires that the upper range of RTs for the single-target trials be greater than that for the doubletarget trials. Accordingly, the slowest 5\% of the RTs were determined separately for each subject for single- and double-target trials under simultaneous-cue and precue conditions. The mean of the subjects' means for these four conditions are shown in Table 3. For precue and simultaneous-cue conditions, the mean of the slowest $5 \%$ of the RTs was greater for the single-target condition. A $t$ test of the mean difference collapsed over cuing condition showed the single-target condition to have a significantly higher range of RTs $[t(3)=3.08, p<.05]$. These results are consistent with a serial processing mixture distribution interpretation of the single-target trial latencies.

\section{Discussion}

Results of the above experiment show a clear and substantial redundancy gain when the double-target conditions are compared with the condition where a target occurred only in one cued location and the other cued location contained the noise letter. Response latency was 
approximately $35 \mathrm{msec}$ less either when the two cued locations contained a repetition of the target letter or when one location contained one target and the other location the alternative target. Grice, Canham, and Gwynne (1984) found a redundancy gain when they compared their double-target condition with a single-target-plus-noise condition. In our Experiment 1, the use of eight-letter displays can be presumed to have saturated a distraction component.

The most important finding from Experiment 1, however, is that the redundancy gain from double targets appears to be independent of whether the same target occurs twice or whether the other cued location is occupied by the alternative target. This finding would appear to pose serious problems for a coactivation hypothesis. One can readily conceive of how processing the letter $S$ on one location on the retina and a separate $S$ on a different location could feed into an identification mechanism with a pooling of the information. It is inconceivable, however, that the identification of the target letter $S$ should be facilitated in this manner by the simultaneous processing of the letter $\mathrm{C}$ on a different retinal locus. Even locating the presumed facilitation of coactivation at the response side would not be a feasible alternative. Any response priming received by the processing of the letter $S$ would only be interfered with by the simultaneous processing of the letter $\mathrm{C}$, which would be priming the alternate response, leading to response competition.

A failure to find evidence for coactivation could be attributed to the use of eight-letter displays. With eight letters presented simultaneously to the visual system, any pooling of information between the processing of two identical letters may well have been masked or lost in the information coming from six different letters. We will address this possible situation in Experiment 2.

The finding that the redundancy gain is the same for the identical- and both-targets conditions is compatible with either a separate activation model or a serial processing of the two cued locations. If we assume that the discrimination required is difficult enough to require some degree of attentional resources and to preclude automatic processing, then a separate-activation model assumes divided attention, while serial processing is consistent with a unitary attentional focus that cannot be simultaneously directed to separated locations in the visual field. We employed the both-targets condition not only to test for coac-

Table 3

Mean of the Maximal Response Times (in milliseconds) for Single- and Double-Targets Conditions Obtained with Simultaneous Cues and 150-msec Precues

Target Conditions

\begin{tabular}{lcc} 
& \multicolumn{2}{c}{ Target Conditions } \\
\cline { 2 - 3 } & $\begin{array}{c}\text { Single Target } \\
\text { and Noise Letter }\end{array}$ & Double Targets \\
\hline Simultaneous Cue & 812 & 750 \\
150-msec Precue & 776 & 702 \\
Combined Mean & 794 & 726 \\
\hline
\end{tabular}

tivation but also to help distinguish between divided and unitary attention.

If attention is simultaneously directed to both cued locations, the parallel processing of the two stimuli would provide a race situation in which the first target to reach a recognition criterion would eventuate in a response. If this was all that was involved, it would make no difference whether the separate targets were identical or represented the two alternatives. However, there is the phenomenon of response competition to consider. In twochoice RT tasks, if a target letter is presented flanked by the alternative target, there is a marked increase in RT. The effect has been interpreted in terms of response competition (e.g., Coles et al., 1985; B. A. Eriksen \& C. W. Eriksen, 1974; C. W. Eriksen \& Schultz, 1979; Flowers \& Wilcox, 1982). Both target and flankers are assumed to be parallel-processed, and the flankers result in incipient activation of the response appropriate to them. This inhibits and slows the execution of the correct response. If the target is flanked by repetitions of itself or responsecompatible stimuli, there is relatively little effect upon target RT.

In Experiment 1, the both-targets condition is comparable to flanking the target with the alternative target, and the identical-targets condition to flanking the target with repetitions of itself. The response-competition effect would seem to predict that the both-targets condition would have appreciably longer RTs than would the identical-targets and single-target conditions. Our failure to find any evidence of response-competition effect in the results of Experiment 1 would suggest that the separate targets in the double-target condition were not processed simultaneously or in parallel.

The response-competition effect has been used to assess the locus of attentional focus in the visual field (C. W. Eriksen \& Hoffman, 1973; C. W. Eriksen \& St. James, 1986; Murphy \& C. W. Eriksen, 1987). These studies have shown that the attentional focus cannot be reduced below about a degree of angle of the visual field and that there is a processing gradient around this focus. The response-competitive effect of an incompatible response noise letter decreases as the noise letter is separated from the target by increasing distances out to about a degree or a degree and a half of visual angle. If sufficient time $(150 \mathrm{msec})$ is allowed for attention to focus upon the target, there is little or no observable effect when the noise is beyond a degree of angle from the target (Murphy \& C. W. Eriksen, 1987). These findings are pertinent to our present discussion since they suggest that response-competitive effects would have been obtained only if visual attention could be divided and directed simultaneously to separated locations in the visual field. The two cued locations in Experiment 1 were always separated by a minimum distance of $1.5^{\circ}$, and, especially in the precued condition, ample time was present for the attentional focus to have been located on the cued location. Thus, the absence of response-competition effects for the 
both-targets condition strongly suggests that the subjects were not dividing their attention on a trial between both cued locations.

The results are well described by a unitary attentional focus that is serially directed to the two cued locations. If a target is found in the first location, the appropriate response is initiated. Trials on which this occurs should have the same minimal latencies for their distribution whether or not the other cued location contained a noise letter, a repetition of the target letter that occurred in the first position searched, or the alternative target letter. In the latter case, response competition would not occur since attention would not be directed to the other cued position containing the alternative target.

With serial search, if a target is not found in the first position, some time presumably will be consumed in processing the noise letter, to the point that a decision is made that it is not a target. Attention would then need to be directed to the second cued location. In Experiment 1, we estimated this time by doubling the mean difference in RT between the single- and double-target conditions. This value was about $70 \mathrm{msec}$, a time that converges well with a comparable estimate for the duration of this mental operation in the C. W. Eriksen and Yeh (1985) experiment. It also is consistent with the search rate reported by Treisman and Gelade (1980). Since attention would not have to be redirected to the second cued location in either double-target condition, mean latency would be shorter in these conditions by half the duration required to recognize noise and redirect attention. Furthermore, the maximal latencies for these double-target conditions would be shorter than those for target-plus-noise trials, expectations that were confirmed in the above analysis of results.

The conclusion from Experiment 1 that the subjects did not divide their attentional resources between the two cued locations is based upon the absence of any suggestion of a response-competition effect in the both-targets condition. C. W. Eriksen and Schultz (1979) and Grice et al. (1982) have interpreted the response-competition effect as a manifestation of continuous flow processing of the response-competitive stimuli. In this conception, information about a visual stimulus is assumed to accumulate gradually over time in the visual system. Partial response activation begins as information begins to accumulate. Early in the process, the information is consistent with a wide range of responses, and these receive initial activation. As information continues to accumulate, response priming becomes increasingly directed to those responses that remain viable alternatives. A given response is evoked when the level of priming in its channel satisfies a criterion. However, the response must be executed against the background of partial activation of these other primed responses. Partial activation of competing responses delays the execution of the correct response.

If the continuous-flow account of response competition is accepted, then the failure to obtain response competition in Experiment 1 is very strong evidence against divided attention in the visual field. However, there is a version of a discrete-stage model that could account for the result and be consistent with a divided-attention interpretation. We will term this a leaky-decisionstage-model.

Consider a version of a race model in which both cued locations are processed in parallel. Processing is fed to a decision stage, and a response is emitted whenever the criterion for that response is reached. In Experiment 1, RT was faster with double targets than it was with a single target, due to statistical facilitation, even when the two targets called for different responses. Responsecompetition effects were avoided in the experimental arrangement of Experiment 1, because it differed from the usual response-competition paradigm in an important way: In the paradigm where response-competition effects are prominent, there is only one acceptable location for the target. Competition effects are obtained by flanking this target position with the incompatible stimuli. If the incompatible stimulus finishes processing first and arrives at the decision stage, response evocation must be inhibited until location information is checked. If the location is not correct, the response must be inhibited, and the decision stage awaits the arrival of a stimulus that can meet the location criterion. If we assume that the decision stage is leaky so that partial priming of the response to the incompatible stimulus occurs, then we have the basis for observed competition effects.

In Experiment 1, both cued positions were acceptable target locations. When targets occupy both positions, whichever target arrives first at the decision stage will pass the location criterion and evoke a response. This will result in the statistical facilitation of RT. Since it eliminates the need to inhibit responses in the decision stage, there will be no partial priming of an inhibited response. Thus, identical- and both-targets conditions would not be expected to differ.

There is strong evidence against this version of a leaky decision stage as the basis for response competition. Experiments in support of the continuous-flow account show partial activation of the competing responses measured by electromyographic activity and by strain gauge measurements (Coles et al., 1985; C. W. Eriksen, Coles, Morris, \& O'Hara, 1985). Also, and perhaps more important in terms of the continuous-processing model, the amount of competing-response activation is found to be proportional to the similarity, or feature, overlap between neutral noise stimuli and incompatible stimuli. Thus, a neutral stimulus (a stimulus that does not have an experimentally defined response) will produce response competition for a target stimulus, to the degree that the neutral stimulus shares features with the alternative target (Miller, 1982b; Yeh \& C. W. Eriksen, 1984). This ability of similar stimuli to partially activate or prime competing responses is consistent with the continuousprocessing model in which response priming continuously occurs as information gradually becomes available. It is not consistent with the discrete-stage model in which the product or processing is passed discretely to a decision 
stage. The product of the processing would be a stimulus for which no response was activated in the context of the experimental situation.

However, the assumption of a leaky decision stage would make a divided-attention explanation for the data of Experiment 1 plausible. Both targets can be processed in parallel without resulting in response competition. Whichever target completes processing first triggers its appropriate response. Experiment 2 was carried out in the hope that further information would help to decide between a unitary- and a divided-attention explanation.

\section{EXPERIMENT 2}

The experimental strategy of Experiment 2 involved presenting a single target letter accompanied by a noise letter that either had features in common with the target letter or with the alternative target that was not presented. If both target and noise letter were simultaneously attended, that is, parallel-processed, it should have made no difference whether the noise letter accompanying a target was similar to the presented target or similar to the alternative target. On trials where the noise letter finished processing before the target letter, identification of the noise letter should not have primed a response. The noise letter had no response associated with it in the context of Experiment 2. Thus, even a leaky decision maker would have had nothing to leak to an associated competitive response system. A unitary attentional focus that serially processed the target and the noise letter would have predicted that RT would be longer when the noise letter shared features with the alternative target. On half of the trials on which attention focused first on the noise letter, continuous-flow processing would have resulted in the priming of the response appropriate to the target that shared features with the noise letter. Response competition was expected to ensue when the noise letter was similar to the alternative target, due to a priming buildup that would still be present when attention was shifted to the target in the display. If the noise letter shared features with the target letter presented on that trial, no conflict would have been present.

In Experiment 2, the task was again two-choice RT with the target letters A and Y. Unlike Experiment 1, displays consisted of either one or two letters. One-letter displays consisted of a single occurrence of one of the two target letters. The two-letter displays were of four kinds: (1) the redundant display in which the target letter was repeated; (2) the both-targets display, which contained both the A and the Y; (3) target with similar noise; and (4) target with dissimilar noise. The noise letters were $\mathrm{H}$ and $\mathrm{V}$, chosen for their similarity with the respective targets. On each trial, one or two letters appeared in any one or two of eight positions on an imaginary circle whose diameter subtended $2^{\circ}$ of visual angle and whose center was located at the fixation point.

\section{Method}

Subjects. Eight University of Illinois undergraduate students (4 men and 4 women) served as subjects and were paid for their participation. All subjects were right-handed and had normal or corrected-to-normal vision.

Apparatus and Procedure. Stimuli were presented on a threefield Scientific Prototype model GA tachistoscope. Luminances of the field were equated at $10 \mathrm{fL}$, as measured by a Spectra spot photometer. One field was used as a stimulus field, while another was used as an adaptation field containing a black fixation cross on a white background. Trials were initiated by the subject via a handheld trigger, following a ready signal from the experimenter. The subjects were instructed to fixate the cross to make sure it appeared sharp and clear before initiating the trial. The duration of each stimulus display was $50 \mathrm{msec}$.

The stimuli were black Futura Demi-Bold letters on a white background. Each letter subtended a visual angle of $.22^{\circ}$ horizontally and $.3^{\circ}$ vertically. Each target letter (A and $\mathrm{Y}$ ) could appear alone, repeated twice in the display (redundant), with each other (both targets), or with either one of the two noise letters ( $\mathrm{H}$ and $\mathrm{V}$ ). Fiftysix trials were generated using each of the two targets for all five trial types. For trial types with two letters, the 56 trials were defined by all possible combinations of positions in the display $(8 \times$ 7). On trials in which a single target appeared alone, the target occurred equally often ( 7 times) in each of the 8 positions. The 56 trials $\times 2$ targets $\times 5$ trial types factorially combined to define the 560 trials performed over three experimental sessions. The experimental sessions were preceded by a practice session in which 100 trials were performed.

The subjects were instructed to indicate which target they saw by moving a lever in one direction for one target and in the opposite direction for the other target. Half of the subjects moved the lever to the left for the target $A$ and to the right for $Y$. The remaining subjects received the reverse target-response assignment. The subjects were instructed that, on trials where both targets occurred, the lever could be moved in either direction. Correct RTs were recorded and reported as feedback for the subjects. Due to a programming error, RTs for incorrect trials were not recorded. However, the subjects were informed of the incorrectness of responses.

\section{Results and Discussion}

Table 4 shows the mean response latencies and mean percent errors for the five conditions of Experiment 2. A within-subjects ANOVA of the latencies showed the difference between conditions to be significant $[F(4,28)$ $=13.73, p<.001]$.

We will examine the data in more detail from the assumption of a unitary-attention focus and the serial

Table 4

Mean Response Times (in milliseconds) for the Different Target Conditions of Experiment 2

\begin{tabular}{|c|c|c|}
\hline $\begin{array}{c}\text { Target } \\
\text { Conditions }\end{array}$ & $\begin{array}{c}\text { Mean } \\
\text { Response } \\
\text { Times }\end{array}$ & $\begin{array}{c}\text { Mean } \\
\text { Percent } \\
\text { Errors }\end{array}$ \\
\hline Single Target & 413 & 6 \\
\hline Identical Targets & 425 & 5.6 \\
\hline Both Targets & 434 & 1.9 \\
\hline \multicolumn{3}{|l|}{ Target and } \\
\hline Similar Noise Letter & 450 & 10.7 \\
\hline \multicolumn{3}{|l|}{ Target and } \\
\hline Dissimilar Noise Letter & 467 & 14.6 \\
\hline
\end{tabular}


processing of the two-letter displays. Within this assumption, no real difference would be expected between singleand double-target displays (identical- and both-targets conditions). With single-target displays, attention would focus on the only letter in the display, the processing of which would lead to a discriminated response. With identical- and both-targets displays, attention would focus on one of the letters. It would be immaterial which letter was focused upon, since processing of either letter in the display would produce a target that would lead to a discriminated response.

The means in Table 4 show that this account is not quite so straightforward. By planned comparisons, performance was significantly faster with the single-letter displays than it was with either of the double-target displays $(p<.05)$. This conforms with the finding of Grice, Canham, and Gwynne (1984), who attributed the decrement in performance with two-letter displays to a distraction component. In their experiment, the comparison was between singleand two-letter displays consisting of a target letter and a noise letter. The decrement in performance with their two-letter display could have been due to the subject's first focusing attention on the noise letter and then having to redirect attention to the target. Thus, a distraction component is confounded with an attentional process.

In Experiment 2, the faster performance with the singleletter displays cannot be attributed to the need to redirect attention on half of the trials. The obtained difference in performance is more directly reflective of the distraction component. Such a pure distraction component in a twochoice visual task is commensurate with other reports in the literature. C. W. Eriksen and Hoffman (1972) and Colegate, Hoffman, and C. W. Eriksen (1973) found that choice RT increased with the number of elements in visual displays even when the location of the target was clearly delineated beforehand. C. W. Eriksen, Hamlin, and Daye (1973) and C. W. Eriksen and Schultz (1978) have reported that, when target letters were always presented directly above the fixation point, the occurrence of irrelevant letters, numbers, or even geometrical forms and patches of color significantly retarded RT to the target. Similar distraction effects have been more recently found by Treisman, Kahneman, and Burkell (1983) and Kahneman, Treisman, and Burkell (1983). The existence of a distraction component is not inconsistent with a unitary attentional focus interpretation of the present finding of the significant difference between single- and doubletarget displays. In fact, it saves the assumption.

A unitary attentional focus predicts that the doubletarget displays would be faster than displays containing a single target and a noise letter. Examination of Table 4 shows that this result was obtained. By planned comparison, the identical- and both-targets conditions were found to significantly differ from both the similar- and dissimilar-noise conditions. In these comparisons, the distraction component is held constant, since all four of these displays contain two letters. However, with the displays containing a similar or dissimilar noise letter along with the target, on half of the trials, the subjects were expected to expend some time redirecting attention from a noise letter to the target.

As in Experiment 1, examination of the fastest RTs for the different conditions can be informative. For each subject, the fastest $5 \%$ of RTs was determined for each of the five experimental conditions. These values were averaged over subjects, and the means are shown in Table 5 for the five conditions. An ANOVA of these values (conditions $\times$ subjects) failed to reveal a significant difference. The failure to find a difference is consistent with the unitary-attention hypothesis. Due to the distraction effect, minimal RTs would be expected to be somewhat less under the single-target condition, but in the other four conditions (all consisting of two-letter displays), the minimal RTs should be the same.

The RT distributions for target-plus-similar-noise and target-plus-dissimilar-noise conditions are assumed to be mixture distributions. They are a mixture of those trials where the subject focused attention first on the noise letter and then had to redirect attention to the other letter and those trials where the subject focused attention first on the target letter. The minimal RTs would be expected to come from the latter distribution, which would be essentially the same as that obtained for the two doubletarget conditions where all RTs were expected to be the result of processing the first letter attended.

Although the overall variation among the means of the minimal RTs was not significant, the trend in the values was both supportive and nonsupportive of the above reasoning. Consistent with the distraction effect, the mean for the single-target condition was the lowest; however, it is somewhat disturbing that the minimal RTs were the highest for the targets-plus-noise conditions. This is suggestive that some other effect might have been operating.

The most important finding in Experiment 2 is the difference in mean correct RTs between target-plussimilar-noise and target-plus-dissimilar-noise conditions. Planned comparisons show that the RT to target-plussimilar-noise was significantly faster than to target-plusdissimilar-noise $(p<.05)$. This finding needs to be considered in relation to the lack of a significant difference between identical- and both-targets conditions. With simi-

Table 5

Mean of the Minimal Response Times (in milliseconds) for the Different Target Conditions in Experiment 1

\begin{tabular}{|c|c|}
\hline $\begin{array}{c}\text { Target } \\
\text { Conditions }\end{array}$ & $\begin{array}{c}\text { Mean } \\
\text { of Minimal } \\
\text { Response Times }\end{array}$ \\
\hline Single Target & 308 \\
\hline Identical Targets & 327 \\
\hline Both Targets & 314 \\
\hline \multicolumn{2}{|l|}{ Target and } \\
\hline Similar Noise Letter & 334 \\
\hline \multicolumn{2}{|l|}{ Target and } \\
\hline Dissimilar Noise Letter & 347 \\
\hline
\end{tabular}


lar and dissimilar noise, we obtained clear evidence of response competition. However, the both-targets condition, as in Experiment 1, revealed no evidence of response competition. In prior research on the response-competition effect, the alternative target has always given substantially more RT interference than has a neutral letter having a high feature overlap with the alternative target (B. A. Eriksen \& C. W. Eriksen, 1974; C. W. Eriksen \& B. A. Eriksen, 1979, C. W. Eriksen \& St. James, 1986).

As we noted in Experiment 1, a separate-activation, or divided-attention, hypothesis would predict an appreciable increase in RT when both target letters were presented in the display, a lesser but still substantial increase in RT should occur when the noise letter similar to the other target was present. In both instances, the continuous-flow processing of the features of the target and of the alternative target would prime incompatible responses. We noted that discrete-stage processing could account for the response-competition effects of the standard paradigm by assuming a leaky decision stage. The use of similar- and dissimilar-noise conditions in Experiment 2 was to test the adequacy of the leaky-decision-stage hypothesis.

In the standard response-competition paradigm, the target occurs in a designated position and is flanked by the alternative target letter. With parallel processing of target and noise, if the alternative target noise letter finishes processing first, the response to it must be inhibited since it doesn't meet the location requirement for target. With a leaky decision stage, processing of the noise letter could lead to incipient priming of the response associated with that letter. In Experiment 2, there was no location requirement for targets. In the both-targets condition, there was no need to inhibit a response while the location was checked. With no inhibited response, there was no leaking of priming to the response system and, therefore, no basis for response competition. Thus, the absence of response competition in the both-targets condition was made compatible with the divided-attention hypothesis.

Divided attention and a leaky decision stage, however, cannot very well accommodate the finding in Experiment 2 that the presence of a noise letter sharing features with the alternative target (dissimilar noise) leads to significantly longer RTs than does the presence of a noise letter sharing features in common with the presented target (similar noise). If the dissimilar noise letter should finish processing before the target, there is no experimentally defined response to be activated for this noise letter. Thus, there is no leaking of a prime by the decision stage to the response for the alternative target. In Experiment 2 , if the target on a trial of the dissimilar-noise condition was $Y$, and the noise letter $H$ reached the decision stage first, there was no reason to activate, or prime, the response for an $\mathrm{A}$, since the letter had been identified as an $\mathrm{H}$ for which no response was relevant.

A unitary attentional focus with serial processing predicts no response competition for the both-targets condition, but the dissimilar-noise condition should produce competition effects. In the both-targets condition, with serial processing, the alternative target is never processed before the response to the first processed letter is initiated. However, in the dissimilar-noise condition, on half of the trials, the noise letter with features similar to the alternative target will be processed first. During processing, the similarity of features will lead to some priming of the response appropriate to the target that the noise letter resembles. Consequently, when attention is directed to the target in these displays, the target response will suffer from inhibition due to the partial activation of the alternative response.

\section{GENERAL DISCUSSION}

Our major concern in these experiments has been to determine the role of focused attention in visual discrimination tasks that employ redundant signals. To ensure the use of focused attention, we used discrimination tasks that in past research have required attention. The results are clear-cut in showing that a redundancy gain is only obtained when the RT to redundant targets is compared with the RT to a single-target-plus-noise stimulus. This result is consistent with the findings of Grice, Canham, and Gwynne (1984). RT to single targets occurring in an otherwise empty display is significantly faster than the RTs obtained to displays containing two occurrences of the target.

Grice, Canham, and Gwynne (1984) attributed the superior performance with a single target to a distraction effect that occurs when two or more stimuli occur in the visual field. As we have noted above, there is considerable support for a distraction effect. C. W. Eriksen and Hoffman (1972) and Colegate, Hoffman, and C. W. Eriksen (1973), for example, found that RT on two-choice discriminations increased directly as the number of irrelevant stimuli in displays increased from 4 to 12 . This effect was present even when the location of the target was clearly designated prior to the presentation of the display.

The results of the present two experiments also show the presence of a distraction effect. More importantly, they also show that the slower RT obtained when a target and a noise letter are presented in the display is, at least in part, due to serial attending to the two stimuli. However, if serial attending was all that was involved, we would expect that double target displays would yield RTs comparable to those obtained with single targets presented without a noise letter. In our Experiment 2 and in the Grice, Canham, and Gwynne (1984) experiments, single-target displays were consistently faster than were double targets. It is here that the distraction component is invoked. The added stimulus in the display, through some still undetermined means, slows performance.

A major innovation in the present experiments was the use of displays in which both targets were presented. The finding that these both-targets displays gave redundancy gains comparable to those obtained with displays containing a repetition of the same target (identical-target displays) enabled us to determine that the stimuli in the dis- 
plays were serially searched, or processed, by the attentional focus. If the two stimuli were parallelprocessed, that is, if attention was divided or allocated simultaneously to separated locations in the visual field, response competition would have been expected to occur for the both-targets displays.

We noted that the absence of response-competition effects could be explained by assuming that they were due to a leaky decision stage rather than continuous-flow priming. However, the results of Experiment 2 convincingly ruled out that possibility. In Experiment 2, responsecompetition effects were obtained when a single target letter was presented along with a noise letter that shared features with the alternative target. As expected, on the basis of response competition, a noise letter that was similar to the presented target gave RTs significantly shorter than when the accompanying noise letter was similar to the alternative target. Separate activation, parallel processing, or divided attention cannot accommodate this result. If the target and noise letter were processed in parallel, the features of the noise letter would indeed prime the response appropriate for the similar target, thus yielding response-competition effects. But if the features of a noise letter were sufficient to prime the response, why weren't response-competition effects obtained with both-targets displays?

The finding of response-competition effects with noise letters similar to targets and the lack of such effects with both-targets displays is accounted for, in a simple straightforward manner, by assuming that the two stimuli in the display are serially processed. With both-targets displays, a unitary attentional focus is directed to one of the stimuli. Whichever stimulus is chosen first as the object of attentional focus results in a target identification. No response competition occurs, since the alternative target in the other display position is never processed or is processed after the response has been initiated for the target processed first. With displays containing a target and a noise letter similar to the other target, on half of the trials, that attentional focus will be directed first to the noise letter. As this noise letter is processed, the features it shares with the other target will prime the response appropriate to this other target. When the noise letter is recognized as a noise letter, attention will shift to the real target in the display. When the target is identified, the response to this target will be initiated in competition with the prime that has occurred due to processing the noise letter first. The competition slows the response.

Our use of both-targets displays has also permitted a test of the coactivation hypothesis. Miller (1982a), among others (e.g., Logan, 1980; Nickerson, 1973), has proposed that separate but simultaneous inputs can pool the results of their processing, leading to an increase in activation of recognition responses or an increase in the level of arousal, and thus speeding the execution of responses.

In experimental tests of coactivation, Miller used the criterion
$\mathrm{P}(\mathrm{RT}<t / \mathrm{S} 1+\mathrm{S} 2) \leq \mathrm{P}(\mathrm{RT}<t / \mathrm{S} 1)+\mathrm{P}(\mathrm{RT}<t / \mathrm{S} 2)$

We feel that this criterion is overly severe. Considerable coactivation could be occurring without even equaling the level predicted by separate-activation models. It has been pointed out (C. W. Eriksen, 1988) that the presence of fast guesses in the RT distributions for single-target trials will overestimate the level predicted by separate activation. Furthermore, the distraction component and a possible division of attentional resources over two or more targets would be expected to reduce the values of P(RT $<t / S 1)$ and $\mathrm{P}(\mathrm{RT}<t / \mathrm{S} 2)$ from those values empirically obtained from single-target trials. If attentional resources are limited, the simultaneous processing of each target on redundant-targets trials would be expected to be slower than would the processing of a target on single-target trials. Unless allowance is made for these contingencies, redundant-target facilitation may not even reach the level predicted by separate-activation models.

Our method of comparing double-target displays with both-targets displays avoids the above problems. In both kinds of displays, the distraction effect is the same, and, if attentional resources can be divided, both displays should result in comparable division. Clearly, the coactivation hypothesis requires that displays containing two identical targets should result in a faster RT than should displays containing one of each of the targets. We should note, however, that a finding of faster RTs for identicaltarget displays would not be unequivocal evidence for coactivation, since response competition could cause the both-targets displays to yield slower RTs. Thus, this comparison is only capable of falsifying the coactivation hypothesis.

In neither of our two experiments was significant evidence of coactivation obtained. In Experiment 1, there was no significant or suggestive difference in mean RT for identical- and both-targets displays. However, the presence of six irrelevant noise letters in the displays might well have masked out any facilitation in processing that would arise from the same target letter being processed at two different locations on the retina. In Experiment 2, where only two letters occurred in the display, identicaltarget displays were indeed faster by 9 msec than bothtargets displays. Although this difference was not significant, it is suggestive and should be followed up by a more statistically powerful experiment.

If coactivation does occur, it is not inconsistent with our interpretation of a unitary attentional focus. Facilitation in processing due to identical simultaneous inputs could occur at very early stages in processing, well before the attentional level or a decision stage is involved.

We would stress that our conclusions in this paper regarding the undivided nature of attention and its role in the problem of redundant signals apply only to tasks that require or can benefit from selective attention. Quite different results might be obtained with discrimination tasks that can be handled with automatic processing or 
by tasks where the redundant stimuli are spaced close enough together to fall in a common attentional focus of about $1^{\circ}$ of angle in extent (C. W. Eriksen \& St. James, 1986; Murphy \& C. W. Eriksen, 1987). Variables such as these may well account for a large part of the conflicting findings in the redundant-signals literature.

\section{REFERENCES}

Broadbent, D. (1982). Task combination and the selective intake of information. Acta Psychologica, 50, 253-290.

Colegate, R. L., Hoffman, J. E., \& ERIKsen, C. W. (1973). Selective encoding from multielement visual displays. Perception \& Psychophysics, 14, 217-224.

Coles, M. G. H., Gratton, G., Bashore, T. R. Eriksen, C. W., \& Donchin, E. (1985). A psychophysiological investigation of the continuous flow model of human information processing. Journal of Experimental Psychology: Human Perception \& Performance, 11, 529-553.

Di LolLo, V. (1977). Temporal characteristics of iconic memory. Nature, 267, 241-243.

DOHERTY, M. E., \& KeEley, S. M. (1969). A Baysian prediction of four-look recognition performance from one-look data. Perception \& Psychophysics, 5, 362-364.

DunCAN, J. (1980). The locus of interference in the perception of simultaneous stimuli. Psychological Review, 87, 272-300.

ERIKSEN B. A., \& ERIKSEN, C. W. (1974). Effects of noise letters upon the identification of a target letter in a nonsearch task. Perception \& Psychophysics, 16, 143-149.

ERIKSEN, C. W. (1966). Independence of successive inputs and uncorrelated error in visual form perception. Journal of Experimental Psychology, 72, 26-35.

ERIKSEN, C. W. (1988). A source of error in attempts to distinguish coactivation from separate activation in the perception of redundant targets. Perception \& Psychophysics, 44, 191-193.

Eriksen, C. W., Coles, M. G. H., Morris, L. R., \& O'Hara, W. P. (1985). An electromyographic examination of response competition. Bulletin of the Psychonomic Society, 23, 165-168.

ERIKSEN, C. W., \& Collins, J. F. (1969). Temporal course of selective attention. Jourmal of Experimental Psychology, 80, 254-261.

ERIKSEN, C. W., \& ErIKSEN, B. A. (1979). Target redundancy in visual search: Do repetitions of the target within the display impair processing? Perception \& Psychophysics, 26, 195-205.

Eriksen, C. W., Hamlin, R. M., \& DAYE, C. (1973). The effect of flanking letters and digits on speed of identifying a letter. Bulletin of the Psychonomic Society, 2, 400-402.

ERIKSEN, C. W., \& HofFMAN, J. E. (1972). Some characteristics of selective attention in visual perception determined by vocal reaction time. Perception \& Psychophysics, 11, 169-171.

ERIKSEN, C. W., \& HoffMan, J. E. (1973). The extent of processing of noise elements during selective encoding from visual displays. Perception \& Psychophysics, 14, 155-160.

ERIKSEN, C. W., \& LAPPIN, J. S. (1965). Internal perceptual system noise and redundancy in simultaneous inputs in form identification. Psychonomic Science, 2, 351-352.

ERIKSEN, C. W., \& SChULTZ, D. W. (1978). Temporal factors in visual information processing. In J. Requin (Ed.), Attention and Performance $V I I$ (pp. 3-23). New York: Academic Press.

ERIKSEN, C. W., \& SchultZ, D. W. (1979). Information processing in visual search: A continuous flow conception and experimental results. Perception \& Psychophysics, 25, 249-263.

ERIKSEN, C. W., \& SPENCER, T. (1969). Rate of information processing in visual perception: Some results and methodological considerations. Journal of Experimental Psychology Monograph, 79(Pt. 2), 116.

Eriksen, C. W., \& ST. JAmES, J. D. (1986). Visual attention within and around the field of focal attention: A zoom lens model. Perception \& Psychophysics, 40, 225-240.
ERIKSEN, C. W., \& YEH, Y.-Y. (1985). Allocation of attention in the visual field. Journal of Experimental Psychology: Human Perception \& Performance, 11, 583-597.

Flowers, J. H., \& WiLcox, N. (1982). The effect of flanking context on visual classification: The joint contribution of interactions at different processing levels. Perception \& Psychophysics, 32, 581-591.

GARDNER, G. T. (1973). Evidence for independent parallel channels in tachistoscopic perception. Cognitive Psychology, 4, 130-155.

Grice, G. R., Canham, L., \& Buroughs, J. M. (1984). Combination rule for redundant information in reaction time tasks with divided attention. Perception \& Psychophysics, 35, 451-463.

Grice, G. R., Canham, L., GWynne, J. W. (1984). Absence of a redundant-signals effect in a reaction time task with divided attention. Perception \& Psychophysics, 36, 565-570.

Grice, G. R., Canham, L., \& Schafer, C. (1982). Development of associative and perceptual interference. Perception \& Psychophysics, 32, 375-387.

Hoffman, J. E. (1979). A two-stage model of visual search. Perception \& Psychophysics, 25, 319-327.

JONIDES, J. (1980). Toward a model of the mind's eye. Canadian Journal of Psychology, 34, 103-112.

JONIDES, J. (1983). Further toward a model of the mind's eye's movement. Bulletin of the Psychonomic Society, 21, 247-250.

Kahneman, D. (1973). Attention and effort. Englewood Cliffs, NJ: Prentice-Hall.

Kaineman, D., Treisman, A., \& Burkell, J. (1983). The cost of visual filtering. Journal of Experimental Psychology, 9, 510-522.

KEELEY, S. M., \& DOHERTY, M. E. (1971). A Bayesian prediction of multiple-look identification performance from one-look data: The effect of unequal prior probabilities. Perception \& Psychophysics, 10, 119-122.

KINCHLA, R. A. (1974). Detecting target elements in multielement arrays: A confusability model. Perception \& Psychophysics, 15, 149-158.

LoGAN, G. (1980). Attention and automaticity in Stroop and priming tasks: Theory and data. Cognitive Psychology, 12, 523-553.

Meruers, L. M. M., \& Eukman, E. G. J. (1977). Distributions of simple RT with single and double stimuli. Perception \& Psychophysics, 22, 41-48.

MILLER, J. (1982a). Divided attention: Evidence for coactivation with redundant signals. Cognitive Psychology, 14, 247-279.

Miller, J. (1982b). Effects of noise letters on decisions: Discrete or continuous flow of information? Perception \& Psychophysics, 31 , 227-236.

Mullin, P. A., Egeth, H. E. , Mordkoff, J. T. (1988). Redundanttarget detection and processing capacity: The problem of positional preferences. Perception \& Psychophysics, 43, 607-610

Murphy, T. D., \& Eriksen, C. W. (1987). Temporal changes in the distribution of attention in the visual field in response to precues. Perception \& Psychophysics, 42, 576-586.

NICKERSON, R. S. (1973). Intersensory facilitation of reaction time: Energy summation or preparation enhancement. Psychological Review, 80, 489-509.

Posner, M. I., Snyder, R. R., \& Davidson, D. J. (1980). Attention and the detection of signals. Journal of Experimental Psychology: General, 109, 160-174.

RAAB, D. (1962). Statistical facilitation of simple reaction time. Transactions of the New York Academy of Sciences, 24, 574-590.

Schneider, W., \& ShIFfrin, R. M. (1977). Controlled and automatic human information processing: I. Detection, search and attention. Psychological Review, 84, 1-66.

ShAw, M. L. (1978). A capacity allocation model for reaction time. Joumal of Experimental Psychology: Human Perception \& Performance, 4, 586-598.

ShaW, M. L., \& SHAW, P. (1977). Optimal allocation of cognitive resources to spatial locations. Journal of Experimental Psychology: Human Perception \& Performance, 3, 201-211.

SHIFFrIN, R. M., \& GARDNER, G. T. (1972). Visual processing capacity and attentional control. Joumal of Experimental Psychology, 93, 72-82. 
Shiffrin, R. M., \& SCHNEIDER, W. (1977). Controlled and automatic human information processing: II. Perceptual learning, automatic attending, and general theory. Psychological Review, 84, 127-190.

Shulman, G. L., Remington, R. W., \& Mclean, J. P. (1979). Moving attention through visual space. Joumal of Experimental Psychology: Human Perception \& Performance, 5, 522-526.

SPERLING, G. (1960). The information available in brief visual presentation. Psychological Monographs, 74, 1-28.

Treisman, A., \& Gelade, G. (1980). A feature-integration theory of attention. Cognitive Psychology, 12, 97-136.

Treisman, A., Kahneman, D., \& Burkell, J. (1983). Perceptual objects and the cost of filtering. Perception \& Psychophysics, 33, 527-532.

Van der Heidden, A. H. C., Schreuder, R., Maris, L., \& Neerincx, M. (1984). Some evidence for correlated separate activation in a simple letter-detection task. Perception \& Psychophysics, 36, 577-585.

YEH, Y.-Y., \& ERIKSEN, C. W. (1984). Name codes and features in the discrimination of letter forms. Perception \& Psychophysics, 36, 225-233.

\section{NOTE}

1. The senior author has examined numerous RT distributions from experiments that have employed choice $\mathrm{RT}$ tasks in which the error rate has been $15 \%$ or less. He has invariably found that both the minima RTs and mean of the error RTs show the same variation over experimental conditions and treatments as do the mean RTs based upon the correct trials. This certainly suggests that fast responses as well as errors are made on the basis of some information and are subject to the same processing effects of the experimental variables as are the correct responses. If true fast guesses do occur, they must be quite rare, at least on tasks where the overall error rate is low.

(Manuscript received July 13, 1988; revision accepted for publication October 14, 1988.) 